\title{
Impact of an Infectious Disease Specialist-Led Postprescription Carbapenem Intervention on Prescribing Behavior in a Japanese Tertiary Hospital: a Before-After Study
}

Takashi MATONO ( $\nabla$ tmatonoh1@aih-net.com )

lizuka Hospital https://orcid.org/0000-0001-9957-0510

Yuichi Umeda

lizuka Hospital

Moritsugu Uchida

lizuka Hospital

Hidenobu Koga

lizuka Hospital

Naoya Kanatani

lizuka Hospital

Yoshimi Furuno

lizuka Hospital

Tomonori Yamashita

lizuka Hospital

Kenichi Nakamura

lizuka Hospital

Research article

Keywords: Infectious disease specialist, antimicrobial stewardship, postprescription carbapenem intervention

Posted Date: April 29th, 2020

DOI: https://doi.org/10.21203/rs.3.rs-20384/v1

License: (c) (i) This work is licensed under a Creative Commons Attribution 4.0 International License.

Read Full License 


\section{Abstract}

Background: There are a few infectious disease (ID) specialists in Japan. A solution to promote the implementation of antimicrobial stewardship activities under these resource-limited settings is required.

Methods: We compared carbapenem consumption between a 24-month baseline and 12-month intervention periods. During the intervention period, an ID specialist provided daily advises through a chart review and ID consultation service to prescribers against all prolonged carbapenem use $(\geq 14$ days). Additionally, we send an aggregated table containing the weekly point prevalence amount of each departmental carbapenem use for 7-13 and $\geq 14$ days through e-mail to all doctors.

Results: Among the 1,241 carbapenem courses during the intervention period, the ID specialist provided a total of 96 instances of feedback regarding carbapenem use for $\geq 14$ days, and the feedback acceptance rate was $76 \%$. After the initiation of the intervention, the trend in monthly carbapenem consumption changed (coefficient: $-0.62 ; 95 \% \mathrm{Cl}:-1.15$ to $-0.087, \mathrm{p}=0.024$ ), and its consumption decreased (coefficient: $-0.098 ; 95 \% \mathrm{Cl}:-0.16$ to $-0.039, \mathrm{p}=0.002$ ) without an increase in the consumption of broad-spectrum antimicrobials or in-hospital mortality. Interestingly, the monthly number of carbapenem courses, but not the duration of carbapenem use, significantly decreased (coefficient: $-3.02 ; 95 \% \mathrm{Cl}:-4.63$ to $-1.42, \mathrm{p}=$ $0.001)$. The carbapenem-related annual estimated savings after the intervention was $\$ 83,745$, with a $22 \%$ cost reduction.

Conclusions: Our ID specialist-led daily intervention with weekly feedback regarding long-term carbapenem use was effective in reducing antimicrobial consumption. Such feedback may be useful in changing the prescribing behavior and promoting appropriate antimicrobial usage even in resourcelimited settings.

\section{Background}

Antimicrobial resistance is a serious global health threat, and the annual antimicrobial resistance-related death is predicted to be 10 million people in 2050 [1]. Given this threatening substantial issue, reducing the use of inappropriate antimicrobials is an urgent task. In acute care hospital, approximately $20-50 \%$ of overall antimicrobials are estimated to be unnecessary or inappropriate [2], and antimicrobial stewardship programs (ASPs) are advocated as key elements in optimizing antimicrobial use in healthcare settings [3]. Among various ASPs, the importance of infectious disease (ID) specialist-led ASPs has been highlighted in recent years [4], involving preprescription authorization (PPA) and postprescription review with feedback (PPRF) as core strategies of ASPs [3, 5]. However, in Japan, there are only a few board-certified ID specialists (1.18 per 100,000 people in Japan versus 2.56 per 100,000 people in the USA) [6-8]; therefore, a lack of human resources is one of the major barriers for the implementation of these evidence-based ASPs. Further, although the Japanese government issued a national action plan on antimicrobial resistance in 2016 , only $14.6 \%$ of overall Japanese medical institutions, including $41 \%$ of hospitals with $>500$ beds, had an approved formal antimicrobial 
stewardship team (AST) in 2018, and most staff members were not full-time equivalent (FTE) [9]. Thus, at present, the Japanese guidance for implementing ASPs has recommended the PPRF strategy instead of the time-consuming PPA [10].

In Japan, third-generation cephalosporin-resistant Enterobacteriaceae has increased in national surveillance data [11]. In our hospital, third-generation cephalosporin-resistant Escherichia coli has been recently identified in approximately $30 \%$ of total $E$. coli cases; therefore, carbapenem consumption increased from a median of 2.19 defined daily doses (DDDs) per 100 patient-days in 2012 to 3.73 DDDs per 100 patient-days in 2016. Despite this alarming increase, we have insufficient protected time to devote to the implementation of PPA and early PPRF, and thus, there is a need for a solution. Hence, the aim of this study was to assess the effectiveness of an ID specialist-led, hospital-based ASP against prolonged carbapenem use in resource-limited settings using weekly feedback and daily intervention.

\section{Methods}

\section{Study design and setting}

This uncontrolled before-after study was designed to compare carbapenem consumption in a 24-month baseline period (from August 2015 to July 2017) to that in a 12-month intervention period (from August 2017 to July 2018). The study was conducted at the lizuka Hospital (AlH), a tertiary care hospital with 1048 inpatient beds and an adult/neonatal intensive care unit in Fukuoka, Japan. The data for this study were obtained via a medical database at the AlH linking a microorganism database in the microbiology laboratory, a prescription database in the pharmacy department, a patient database from the AST, and a chart review.

This study was approved by the Institutional Review Board at the AlH (approval no. 17183) and conducted according to the principles expressed in the Declaration of Helsinki. The need for informed consent was waived because this study only used data collected from clinical practice.

\section{Feedback and intervention}

AST members included a physician, clinical pharmacist, nurse, and microbiology laboratory technician who performed ASP duties since April 2015. We restricted antimicrobial prescription of daptomycin, linezolid, and tedizolid as PPA but did not restrict against carbapenem prescription. Every weekday during the baseline period, two ID-trained pharmacists performed a prospective audit for all carbapenem prescription from the initiation to discontinuation of the prescription with a FTE of 1.1. They predominantly provided dosing optimization feedback to carbapenem prescribers through the patient charts. Moreover, they recorded a template document in the patient charts on the day of carbapenem prescription initiation and after use for 7 days, recommending the proper use of antimicrobials. Indication and duration of carbapenem use was up to the prescribing doctors because their feedback contained neither an alternative prescription choice nor a suggestion for discontinuation. 
During the intervention period, we additionally initiated an intervention by a board-certified ID specialist against all carbapenem use for $\geq 14$ days. The ID specialist provided daily ID consultation through faceto-face or telephone conversations with prescribers and recorded the recommendation in the patient's chart. In addition, we reported an aggregated table involving the weekly point prevalence surveillance indicating the amount of departmental carbapenem use for 7-13 days and $\geq 14$ days through e-mail to all medical doctors at the AlH.

\section{Outcome measure and definition}

The primary endpoint of this study was the trend in monthly carbapenem consumption. Secondary endpoints were 1) change in each intravenous antimicrobial consumption which was available at the AlH, and 2) the trend in the detection of hospital-acquired microorganisms/infections including methicillinresistant Staphylococcus aureus (MRSA), extended-spectrum beta-lactamase (ESBL)-producing Enterobacteriaceae, carbapenem-resistant organisms (CROs), Candida spp. bloodstream infections, and Clostridioides difficile infections (CDI). Additionally, we extracted data for all-cause in-hospital mortality and length of hospital stay from the medical database at the AlH.

Monthly aggregated hospital antimicrobial consumption was measured as days of therapy (DOTs) per 100 patient-days and DDDs per 100 patient-days. The incidence of microorganism detection was calculated as the number of clusters per 1000 patient-days. A microorganism recovered from a previously enrolled patient (e.g., continuous bacteremia) within the same month was excluded. Hospital-acquired microorganism/infection was defined as a microorganism/infection identified $>48$ hours after admission. ESBL-producing Enterobacteriaceae, including Escherichia coli, Klebsiella pneumoniae, Klebsiella oxytoca, and Proteus mirabilis was identified using the Cica $\beta$-test (Kanto Chemical Co., Tokyo, Japan) and the disc diffusion method. CRO was defined as an imipenem-resistant organism based on the Clinical Laboratory Standard Institute (CLSI) M100-S29. CDI was defined as a patient with diarrhea and a stool specimen positive for glutamate dehydrogenase plus toxin assay (C. DIFF QUIK CHEK COMPLETE from 2015-2017; Alere Medical Co., Tokyo, Japan, and GE Test Immunochromato-CD GDH/TOX from 2017-2018; Nissui Pharmaceutical Co., Tokyo, Japan).

The acceptance rate was defined as the number of feedback accepted by prescribers divided by the total number of feedback made by an ID specialist. Acceptance or nonacceptance was decided by chart review within 24 hours after the recommendations were made. A carbapenem-related annual cost reduction and estimated savings were calculated based on the annual cost of purchasing carbapenem. The cost of purchasing carbapenem was adjusted to the cost of branded meropenem in 2018 considering fluctuations in drug prices and cost differences between brands and generics. The exchange rate of 1 USD to 110 JPY in 2019 was used.

\section{Statistical analysis}

We used segmented regression analysis of an interrupted time series to evaluate trends in monthly antimicrobial consumption calculated by DOTs per 100 patient-days and trends in the incidence of detected microorganisms, which was represented as a change in level due to the initiation of the 
intervention and change in trend between two periods [12]. The monthly antimicrobial consumption using DDDs per 100 patient-day, number of detected hospital-acquired microorganisms/infections, all-cause inhospital mortality, and length of hospital stay were compared between the two periods using the chisquared $\left(\chi^{2}\right)$ test for nominal variables and Mann-Whitney $U$ test for continuous variables. A two-tailed $p$ value of $<0.05$ was regarded as statistically significant, and the $95 \%$ confidence interval $(95 \% \mathrm{Cl})$ was used. All analyses were performed using Stata/SE v. 14.2 (StataCorp, College Station, TX, USA).

\section{Results}

\section{Impact of the intervention on carbapenem consumption}

During the study period, 90,076 patients were admitted to the AlH, including 59,195 in the baseline period and 30,881 in the intervention period. Out of these, 3,935 patients received carbapenem (meropenem and doripenem) injection: 2,694 in the baseline period and 1,241 in the intervention period. Among the 1,241 courses of carbapenem in the intervention period, an ID specialist provided a total of 96 instances of feedback against carbapenem use for $\geq 14$ days. The predominant content of the feedback was carbapenem discontinuation $(n=45)$, followed by further investigation $(n=25)$, and change to other antimicrobials $(n=22)$ (Table 1$)$. The overall acceptance rate of the ID specialist's feedback was $76 \%$, and the ID specialist devoted an average FTE of 0.16 to the intervention. After the initiation of the intervention, the trend in monthly carbapenem consumption (DOTs per 100 patient-days) changed in level (coefficient: $-0.62 ; 95 \% \mathrm{Cl}:-1.15$ to $-0.087, \mathrm{p}=0.024$, Fig. 1 ), and its consumption decreased (coefficient: $-0.098 ; 95 \%$ $\mathrm{Cl}:-0.16$ to $-0.039, p=0.002$ ). Interestingly, the monthly number of courses of carbapenem significantly decreased (Fig. 2), changing in level (coefficient: $-18.6 ; 95 \% \mathrm{Cl}$ : -34.2 to $-3.05, \mathrm{p}=0.021$ ) and in trend (coefficient: $-3.02 ; 95 \% \mathrm{Cl}:-4.63$ to $-1.42, \mathrm{p}=0.001$ ). However, the trend in monthly average duration of carbapenem use did not significantly decrease (coefficient: $-0.033 ; 95 \% \mathrm{Cl}:-0.16$ to $0.095, \mathrm{p}=0.61$ ). The adjusted annual costs of purchasing carbapenem in the latest baseline period (from August 2016 to July 2017) and in the intervention period (from August 2017 to July 2018) were $\$ 386,861$ and $\$ 303,116$, respectively. Namely, the carbapenem-related annual estimated savings after the intervention was $\$ 83,745$, with a cost reduction of $22 \%$. There was no change in in-hospital mortality (3.8\% versus $3.7 \%, p$ $=0.53$ ) or length of hospital stay (median 14.5 [interquartile range (IQR) 14.2-14.8] versus 14.3 [IQR $14.0-14.6]$ days, $p=0.52$ ) between the baseline and intervention periods. 
Table 1

Content and acceptance rate of feedback made by an ID specialist against carbapenem use for $\geq 14$ days

\begin{tabular}{|llll|}
\hline Recommendations $(\mathbf{n}=\mathbf{9 6})$ & Intervention $(\mathbf{n})$ & Acceptance $(\mathbf{n})$ & Acceptance rate $(\%)$ \\
\hline Carbapenem discontinuation & 45 & 37 & 82 \\
\hline Further investigation & 25 & 16 & 64 \\
\hline Change to other antimicrobials & 22 & 17 & 77 \\
\hline Source control & 3 & 2 & 67 \\
\hline Dosing optimization & 1 & 1 & 100 \\
\hline Total & 96 & 73 & 76 \\
\hline
\end{tabular}

\section{Change in monthly intravenous antimicrobial consumption}

Comparing the two periods, monthly overall intravenous antimicrobial consumption increased after the intervention (median 37.1 [IQR 35.6-40.8] versus 41.4 [IQR 40.8-42.4] DDDs/100 patient-days, $p=0.02$ ), whereas monthly carbapenem consumption decreased (median 2.28 [IQR 2.04-2.58] versus 1.79 [IQR $1.66-2.02] \mathrm{DDDs} / 100$ patient-days, $p<0.001$, Table $\mathrm{S} 1$ ). Among all available intravenous antimicrobials at the $\mathrm{AlH}$, consumption of piperacillin-tazobactam (median 1.90 [IQR 1.72-2.21] versus 2.32 [IQR 2.032.56] DDDs/100 patient-days, $p=0.002$ ), first-generation cephalosporins (median 2.41 [IQR 2.21-2.80] versus 3.24 [IQR 3.08-3.35] DDDs/100 patient-days, $p<0.001$ ), and third-generation cephalosporins (median 1.72 [IQR 1.63-2.17] versus 2.34 [IQR 2.18-2.46] DDDs/100 patient-days, $p<0.001$ ) predominantly increased after the intervention. There was no significant increase in the consumption of fourth-generation cephalosporins $(p=0.097)$ and fluoroquinolones $(p=0.42)$.

The interrupted time series analysis showed that the trend in monthly piperacillin-tazobactam consumption temporarily increased after the initiation of the intervention, but the level change was not significant (coefficient: $0.25 ; 95 \% \mathrm{Cl}:-0.30$ to $0.79, \mathrm{p}=0.36$, Fig. 3 ). Thereafter, the trend in piperacillintazobactam consumption decreased (coefficient: $-0.094 ; 95 \% \mathrm{Cl}:-0.17$ to $-0.017, \mathrm{p}=0.019$ ). The monthly cefepime consumption did not change in level (coefficient: $-0.080 ; 95 \% \mathrm{Cl}:-0.10$ to $0.26, p=0.37$ ) or in trend (coefficient: $-0.013 ; 95 \% \mathrm{Cl}:-0.037$ to $0.010, p=0.25$ ).

\section{Change in hospital-acquired microorganisms/infections}

Hospital-acquired MRSA and ESBL-producing Enterobacteriaceae were more frequently detected in the intervention period than in the baseline period $(p=0.001$ and $p<0.001$, respectively) (Table 2). However, after the initiation of the intervention, the trend in monthly incidence of MRSA (coefficient: $-0.023 ; 95 \% \mathrm{Cl}$ : -0.069 to $0.023, p=0.32$ ) and ESBL-producing Enterobacteriaceae (coefficient: $-0.0048 ; 95 \% \mathrm{Cl}:-0.027$ to $0.018, p=0.66$ ) did not significantly change (Fig. S1). The CROs, Candida spp. bloodstream infections, and CDI were detected with similar frequency between the two periods (Table 2). 
Table 2

Detection of hospital-acquired microorganisms/infections and all-cause in-hospital mortality among hospitalized patients before and after the intervention

\begin{tabular}{|c|c|c|c|}
\hline & $\begin{array}{l}\text { Baseline period }(n= \\
59195)\end{array}$ & $\begin{array}{l}\text { Intervention period }(\mathrm{n}= \\
30881)\end{array}$ & $\begin{array}{l}\text { p- } \\
\text { value }\end{array}$ \\
\hline In-hospital death, n (\%) & $2247(3.8)$ & $1146(3.7)$ & 0.53 \\
\hline MRSA, n (\%) & $714(1.21)$ & $452(1.46)$ & 0.001 \\
\hline $\begin{array}{l}\text { ESBL-producing Enterobacteriaceae, } \\
\mathrm{n}(\%)\end{array}$ & $323(0.55)$ & $302(0.98)$ & $<.001$ \\
\hline CROs', n (\%) & $124(0.21)$ & $65(0.21)$ & 1.00 \\
\hline $\begin{array}{l}\text { Candida spp. bloodstream } \\
\text { infections, } \mathrm{n}(\%)\end{array}$ & $32(0.054)$ & $19(0.062)$ & 0.66 \\
\hline $\mathrm{CDI}^{*}, \mathrm{n}(\%)$ & $145(0.24)$ & $94(0.30)$ & 0.102 \\
\hline \multicolumn{4}{|c|}{ 'CRO was defined as an imipenem-resistant organism based on the CLSI M100-S29. } \\
\hline \multicolumn{4}{|c|}{$\begin{array}{l}\text { *CDI was defined as a patient having diarrhea with positive for glutamate dehydrogenase plus toxin } \\
\text { assay. }\end{array}$} \\
\hline \multicolumn{4}{|c|}{$\begin{array}{l}\text { MRSA, methicillin-resistant Staphylococcus aureus; ESBL, extended-spectrum beta-lactamase; CROs, } \\
\text { carbapenem-resistant organisms; CDI, Clostridioides difficile infection }\end{array}$} \\
\hline
\end{tabular}

\section{Discussion}

In this before-after study, we evaluated the impact of an ID specialist-led daily postprescription carbapenem intervention with weekly feedback for long-term carbapenem users. Our intervention was effective in reducing carbapenem consumption without causing a change in in-hospital mortality or an increase in the consumption of alternate broad-spectrum antimicrobials. We found that the number of courses of carbapenem, but not the duration of carbapenem use, decreased, indicating a possible beneficial effect of our intervention on improving prescribing behavior.

The present study has three important findings. First, it is noteworthy that our study demonstrates the positive effect of our ASP even in a resource-limited setting. There is limited information on in-hospital ASP in Asia, and resources and settings are diverse among each country and hospital $[9,13,14]$. In Japan, the resources were generally insufficient to meet the demands for implementing an ideal ASP [15], and therefore there is a necessity in developing ID-trained pharmacists and ID specialists [9]. Although PPRF is a well-established intervention, there are few resource-rich Japanese hospitals implementing early PPRF against overall antimicrobial use for $48-72$ hours. Therefore, we must make a practical plan to implement the stewardship according to available institutional resources: e.g., focusing on broadspectrum antimicrobials and/or longer duration of use, and providing once-weekly intervention [16-19]. In our hospital, the number of ID physicians was particularly insufficient; there were no ID fellows, and one (part time) and two (full time and part time) ID specialists worked in the baseline and intervention 
periods, respectively. As a result, only an FTE of 0.16 was devoted to the additional stewardship activity by an ID specialist; however, it is noteworthy that our strategy had a positive effect on reducing carbapenem consumption. Hence, we highlight the positive impact of our ASP strategy, which may be a reasonable and acceptable alternative, particularly in resource-limited settings.

Second, our study indicates that a predominant factor in the decrease of carbapenem consumption might be in behavior change among prescribers. In our study, ESBL-producing Enterobacteriaceae strains were more frequently identified in the intervention period than in the baseline period, a trend similar to that seen in national and local surveillance data in Japan [11]. Therefore, it is meaningful that carbapenem consumption decreased in the intervention period, despite being one of the first-line agents for ESBLproducing Enterobacteriaceae. Interestingly, although we did not provide alternate empirical antimicrobial choices or restrict carbapenem prescription, empirical carbapenem use decreased, which suggests that our intervention was indirectly or comprehensively relevant to changing prescribing behavior. Prescription trends of all available intravenous antimicrobials at our facility showed that the monthly trend in piperacillin-tazobactam consumption increased only temporarily before decreasing, but the change in level was not significant after the initiation of the intervention. There was no compensatory increase in the other antipseudomonal agents including fourth-generation cephalosporins, fluoroquinolones, and aminoglycosides (Table S1). Further, carbapenem-sparing agents such as ceftazidime-avibactam and ceftolozane-tazobactam were not available in our hospital during the study period. As a result, the comprehensive evaluation enables us to know that our intervention might promote optimal use of antimicrobials-that is, reduce unnecessary empirical carbapenem use and using narrow-spectrum antimicrobials such as first- and third-generation cephalosporins-without a change in in-hospital mortality. Thus, we emphasize that not only a reduction in carbapenem consumption but also the positive impact of ASPs on optimizing the use of antimicrobials; the concepts supported by several previous reports $[19,20]$.

Third, our study suggests that, in addition to intervention made by the ID specialist, several factors comprehensively affected the reduction in carbapenem consumption and optimal use of antimicrobials. We only provided direct intervention for $96(7.7 \%)$ of 1,241 courses of carbapenem, and the duration of carbapenem use was not influenced by this. Similar to our weekly feedback, weekly e-mail-based notification alone failed to show a positive impact on reducing carbapenem consumption in a previous study [19]. However, by adding the weekly feedback to the daily intervention on carbapenem use for $\geq 14$ days, synergistic positive effects and a competitive mindset among departments may occur. Further, a hospital president approved our intervention and announced the importance of the intervention and information on antimicrobial resistance to all medical staff at the facility before the initiation of our intervention. The credit and education might yield a further positive influence on growing awareness problems on antimicrobials and changing prescribing behavior. Moreover, leadership of ID consultation services changed from hospitalists to the ID specialist since April 2017 (four month before the initiation of our intervention). We conducted several in-hospital notifications to increase the use of ID consultation services. The campaign and daily ID consultation service without ASP activities might achieve a positive impact on optimal use of antimicrobials. We believe that, in addition to daily intervention, the weekly 
feedback, president's credit, education of medical staff, and campaigns of using ID consultation services all comprehensively and synergistically contributed to a reduction in carbapenem consumption and optimal use of antimicrobials. Hence, we believe that taking action is important without giving up ASP activities in resource-limited settings.

This study has several limitations. First, this is a single-center study in Japan, and thus, it is uncertain whether our hospital-based ASP strategy can be applicable worldwide. Schweitzer et al described that only $48 \%$ and $23 \%$ of studies evaluating antimicrobial stewardship interventions reported clinical and microbiological outcomes, respectively [21]. However, we evaluated these outcomes, and it is noteworthy that our ASP strategy led to a decrease in carbapenem consumption with prescribing behavior change in a resource-limited setting. Second, we could not investigate the length of hospital stay among patients with infectious diseases and infectious disease-related total medical cost reduction. However, we would like to emphasize the impact on cost reduction that our ASP accomplished on overall intravenous antimicrobial-related saving of $\$ 255,352$ in addition to annual estimated carbapenem-related savings $(\$ 83,745)$ on the basis of the annual purchasing cost. Third, there is evidence that the implementation of hospital-based ASP had a positive influence on microbiological outcomes [13, 22, 23]; however, our study did not show the positive microbiological impact on the hospital-acquired multidrug-resistant pathogens and infections. This may be because of the insufficient number of events and short observation period. Further, several studies showed a synergistic effect of hand-hygiene improvement combined with ASP on microbiological outcomes in the hospital $[14,24]$. Thus, we should promote the implementation of hospital-based ASP with hand-hygiene intervention as well as a local-regional ASP to improve microbiological outcomes, and continue further outcome evaluation.

This before-after study showed the positive impact of an ID specialist-led ASP on reductions in carbapenem consumption and antimicrobial-related cost without adversely affecting the increase of broad-spectrum antimicrobial consumption and in-hospital mortality. We believe that an important path to success in hospital-based ASP is investigating institutional/local issues of antimicrobial prescription and making a practical plan according to available institutional resources. In particular, the favorable and reliable relationship between prescribers and the AST is vital in bringing about high compliance and prescribing behavior change among prescribers, which can finally lead to optimal use of antimicrobials. Even in resource-limited settings, we advocate that taking any action is warranted even if it is not PPA and early PPRF.

\section{Abbreviations}

ID:infectious disease; ASP:antimicrobial stewardship program; PPA:preprescription authorization; PPRF:postprescription review with feedback; AST:antimicrobial stewardship team; FTE:full-time equivalent; DDDs:defined daily doses; AlH:lizuka Hospital; MRSA:methicillin-resistant Staphylococcus aureus; ESBL:extended-spectrum beta-lactamase; CRO:carbapenem-resistant organism; CDI:Clostridioides difficile infections; DOTs:days of therapy; CLSI:Clinical Laboratory Standard Institute; IQR:interquartile range 


\section{Declarations}

\section{Acknowledgments}

We would like to acknowledge the physicians, pharmacists, nurses, microbiology laboratory technicians, and other staff at the AlH for their excellent work.

\section{Author contributions}

TM devised the original idea for the study. TM, YU, MU, NK, and TY collected the data. TM and HK led the analysis. TM drafted the original manuscript. All authors contributed to the study design, interpretation of the data, and revision of the manuscript for intellectual content. All authors have read and approved the final version.

\section{Funding}

This study was supported in part by the AlH research grants (grant no. 17183). The funder had no role in this study such as study design, data collection, or decision to publish.

\section{Availability of data and materials}

The dataset analyzed during the current study are available from the corresponding author on reasonable request.

\section{Ethics approval and consent to participate}

This study was approved by the Institutional Review Board at the AlH (approval no. 17183) and conducted according to the principles expressed in the Declaration of Helsinki. The need for informed consent was waived because this study only used data collected from clinical practice.

\section{Consent for publication}

Not applicable.

\section{Competing interests}

All authors declare no conflicts of interest. 


\section{References}

1. O'Neill J. Tackling drug-resistant infections globally. Final report and recom- mendations. Review on Antimicrobial Resistance, May 2016. https://amr-review.org. Accessed September 7, 2019.

2. Schuts EC, Hulscher M, Mouton JW, et al. Current evidence on hospital antimicrobial stewardship objectives: a systematic review and meta-analysis. Lancet Infect Dis. 2016;16:847-56.

3. Barlam TF, Cosgrove SE, Abbo LM, et al. Implementing an antibiotic stewardship program: guidelines by the Infectious Diseases Society of America and the Society for Healthcare Epidemiology of America. Clin Infect Dis. 2016;62:e51-77.

4. Ostrowsky B, Banerjee R, Bonomo RA, et al. Infectious diseases physicians: leading the way in antimicrobial stewardship. Clin Infect Dis. 2018;66:995-1003.

5. Tamma PD, Avdic E, Keenan JF, et al. What is the more effective antibiotic stewardship intervention: preprescription authorization or postprescription review with feedback? Clin Infect Dis. 2017;64:53743.

6. The Japanese Association for Infectious Diseases. Specialists. [in Japanese]. http://www.kansensho.or.jp/modules/senmoni/index.php?content_id=29. Accessed December 31, 2019.

7. American Board of Internal Medicine. Number of Candidates Certified. https://www.abim.org/about/statistics-data/candidates-certified.aspx. Accessed December 31, 2019.

8. The World Bank. Total Population. https://data.worldbank.org/indicator/SP.POP.TOTL. Accessed December 31, 2019.

9. Maeda M, Muraki Y, Kosaka T, et al. The first nationwide survey of antimicrobial stewardship programs conducted by the Japanese Society of Chemotherapy. J Infect Chemother. 2019;25:83-8.

10. The Eight Societies Joint Committee for enhancement of antimicrobial stewardship. Guidance for implementing an antimicrobial stewardship program in Japan. [in Japanese]. Jpn J Chemother. 2017;65:650-87.

11. Japan Nosocomial Infections Surveillance (JANIS). [in Japanese]. https://janis.mhlw.go.jp. Accessed September 7, 2019.

12. Wagner AK, Soumerai SB, Zhang F, Ross-Degnan D. Segmented regression analysis of interrupted time series studies in medication use research. J Clin Pharm Ther. 2002;27:299-309.

13. Honda H, Ohmagari N, Tokuda Y, Mattar C, Warren DK. Antimicrobial stewardship in inpatient settings in the Asia Pacific Region: a systematic review and meta-analysis. Clin Infect Dis. 2017;64:119-26.

14. Apisarnthanarak A, Kwa AL, Chiu CH, et al. Antimicrobial stewardship for acute-care hospitals: an Asian perspective. Infect Control Hosp Epidemiol. 2018;39:1237-45.

15. Doernberg SB, Abbo LM, Burdette SD, et al. Essential resources and strategies for antibiotic stewardship programs in the acute care setting. Clin Infect Dis. 2018;67:1168-74. 
16. Hagiwara D, Sato K, Miyazaki M, et al. The impact of earlier intervention by an antimicrobial stewardship team for specific antimicrobials in a single weekly intervention. Int J Infect Dis. 2018;77:34-9.

17. Fukuda T, Watanabe H, Ido S, Shiragami M. Contribution of antimicrobial stewardship programs to reduction of antimicrobial therapy costs in community hospital with 429 Beds-before-after comparative two-year trial in Japan. J Pharm Policy Pract. 2014;7:10.

18. Honda H, Murakami S, Tagashira Y, et al. Efficacy of a postprescription review of broad-spectrum antimicrobial agents with feedback: a 4-year experience of antimicrobial stewardship at a tertiary care center. Open Forum Infect Dis. 2018;5:ofy314.

19. Akazawa T, Kusama Y, Fukuda $\mathrm{H}$, et al. Eight-year experience of antimicrobial stewardship program and the trend of carbapenem use at a tertiary acute-care hospital in Japan-the impact of postprescription review and feedback. Open Forum Infect Dis. 2019;6:ofz389.

20. Abdallah M, Badawi M, Amirah MF, et al. Impact of carbapenem restriction on the antimicrobial susceptibility pattern of Pseudomonas aeruginosa isolates in the ICU. J Antimicrob Chemother. 2017;72:3187-90.

21. Schweitzer VA, van Heijl I, van Werkhoven $\mathrm{CH}$, et al. The quality of studies evaluating antimicrobial stewardship interventions: a systematic review. Clin Microbiol Infect. 2019;25:555-61.

22. Davey P, Marwick CA, Scott CL, et al. Interventions to improve antibiotic prescribing practices for hospital inpatients. Cochrane Database Syst Rev. 2017;2:CD003543.

23. Karanika S, Paudel S, Grigoras C, Kalbasi A, Mylonakis E. Systematic review and meta-analysis of clinical and economic outcomes from the implementation of hospital-based antimicrobial stewardship programs. Antimicrob Agents Chemother. 2016;60:4840-52.

24. Baur D, Gladstone BP, Burkert F, et al. Effect of antibiotic stewardship on the incidence of infection and colonisation with antibiotic-resistant bacteria and Clostridium difficile infection: a systematic review and meta-analysis. Lancet Infect Dis. 2017;17:990-1001.

\section{Figures}




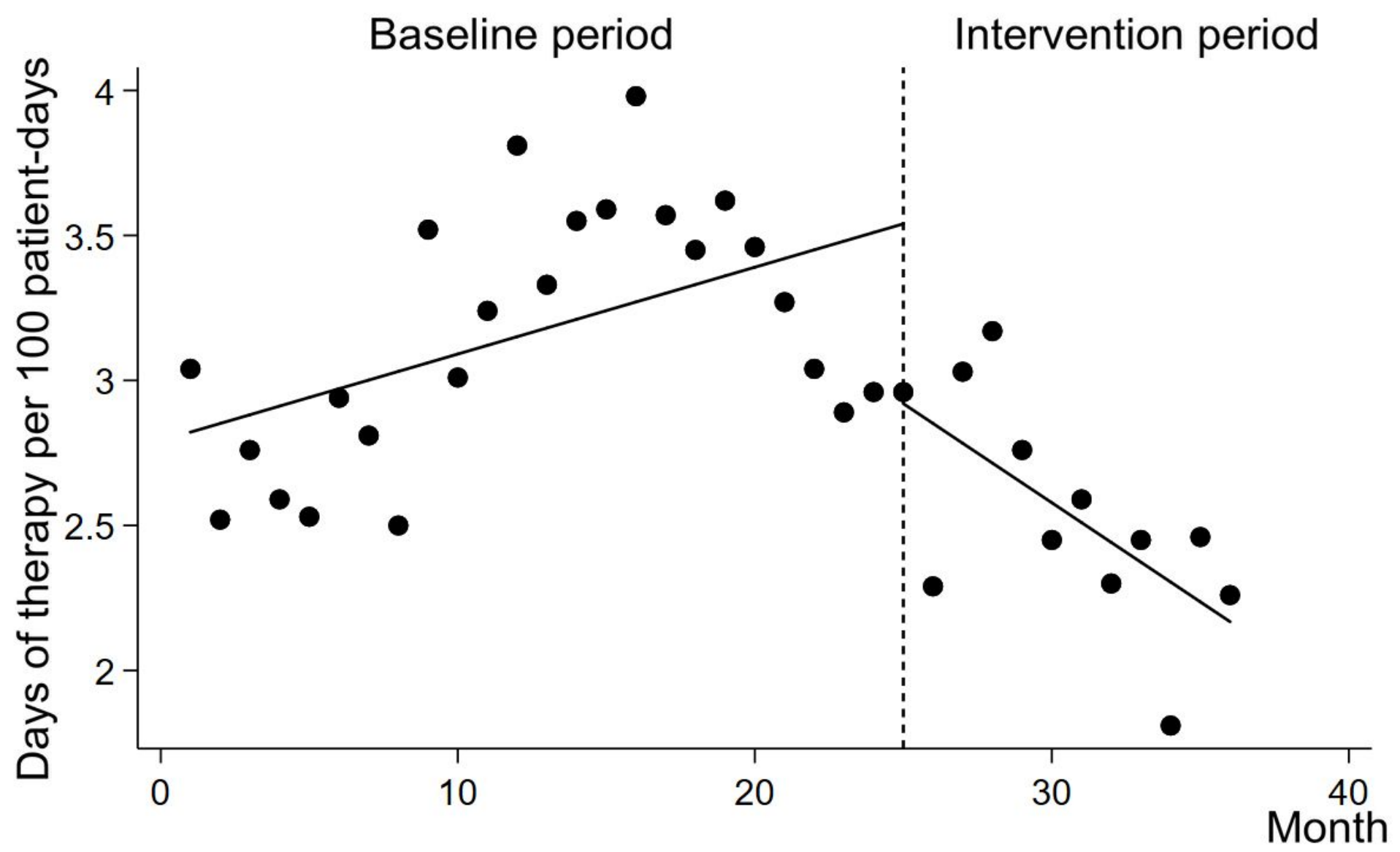

Figure 1

Monthly trend of carbapenem consumption before and after the initiation of the intervention. The monthly trend of carbapenem consumption changed in level (coefficient: $-0.62 ; 95 \% \mathrm{Cl}:-1.15$ to $-0.087, \mathrm{p}=$ 0.024 ) and its consumption decreased (coefficient: $-0.098 ; 95 \% \mathrm{Cl}:-0.16$ to $-0.039, \mathrm{p}=0.002$ ) after the initiation of the intervention.
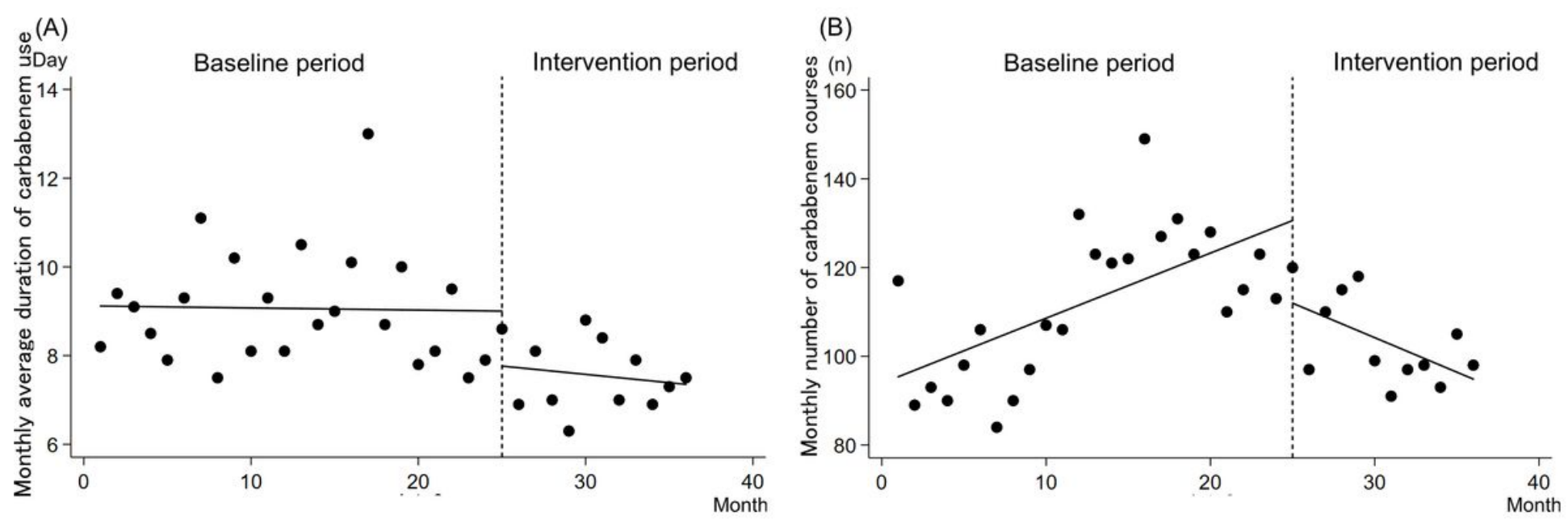

Figure 2 
Monthly trend in average duration of carbapenem use (A) and the number of courses of carbapenem (B). (A) The monthly average duration of carbapenem use did not significantly change in level (coefficient: $-1.24 ; 95 \% \mathrm{Cl}:-2.73$ to $0.26, \mathrm{p}=0.10$ ) or in trend (coefficient: $-0.033 ; 95 \% \mathrm{Cl}:-0.16$ to $0.095, p=0.61$ ). (B) The monthly number of courses of carbapenem decreased after the initiation of the intervention including changes in level (coefficient: $-18.6 ; 95 \% \mathrm{Cl}$ : -34.2 to $-3.05, p=0.021$ ) and in trend (coefficient: $-3.02 ; 95 \%$ Cl: -4.63 to $-1.42, p=0.001)$.
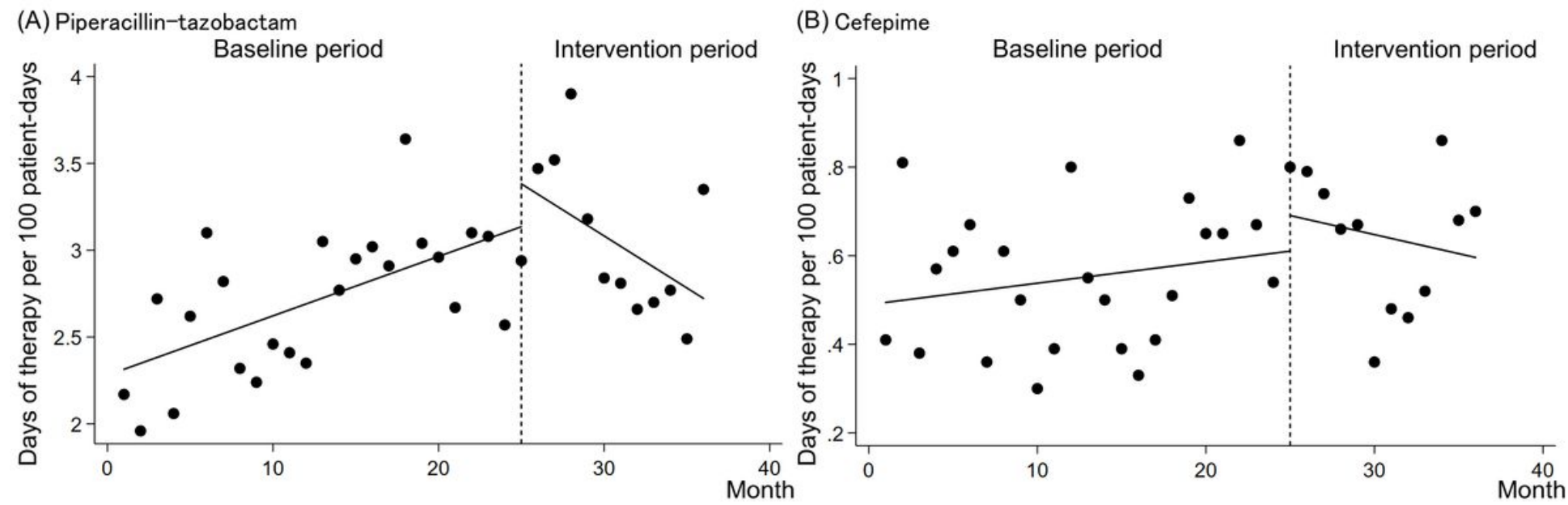

\section{Figure 3}

Trend in monthly piperacillin-tazobactam (A) and cefepime (B) consumption before and after the initiation of the intervention. (A) The trend in monthly piperacillin-tazobactam consumption temporarily increased but the change in level was not significant (coefficient: $0.25 ; 95 \% \mathrm{Cl}:-0.30$ to $0.79, \mathrm{p}=0.36$ ). Thereafter, the trend in piperacillin-tazobactam consumption decreased (coefficient: $-0.094 ; 95 \% \mathrm{Cl}:-0.17$ to $-0.017, p=0.019$ ). (B) The monthly cefepime consumption did not change in level (coefficient: -0.080 ; $95 \% \mathrm{Cl}:-0.10$ to $0.26, p=0.37$ ) or in trend (coefficient: $-0.013 ; 95 \% \mathrm{Cl}:-0.037$ to $0.010, p=0.25$ ).

\section{Supplementary Files}

This is a list of supplementary files associated with this preprint. Click to download.

- FigureS1.jpg

- TableS1.docx 